\title{
THE THRESHOLD FOR COMBS IN RANDOM GRAPHS
}

\author{
JEFF KAHN, EYAL LUBETZKY, AND NICHOLAS WORMALD
}

\begin{abstract}
For $k \mid n$ let $\operatorname{Comb}_{n, k}$ denote the tree consisting of an $(n / k)$-vertex path with disjoint $k$-vertex paths beginning at each of its vertices. An old conjecture says that for any $k=k(n)$ the threshold for the random graph $\mathcal{G}(n, p)$ to contain $\operatorname{Comb}_{n, k}$ is at $p \asymp \frac{\log n}{n}$. Here we verify this for $k \leq C \log n$ with any fixed $C>0$. In a companion paper, using very different methods, we treat the complementary range, proving the conjecture for $k \geq \kappa_{0} \log n$ (with $\kappa_{0} \approx 4.82$ ).
\end{abstract}

\section{INTRODUCTION}

Write $G=\mathcal{G}(n, p)$ for the usual random graph on $V:=[n]:=\{1, \ldots, n\}$, in which edges are present independently, each with probability $p$. We are interested in understanding when (i.e. for what $p$ ) $G$ is likely to contain (a copy of) a fixed $n$-vertex tree $T$.

(Formally we may define the "threshold" for containing $T$ to be that (unique) $p$ for which the probability that $G$ contains $T$ is $1 / 2$. To stay closer to the usual threshold language of [7], or e.g. [10], we would need to work with a sequence $\left\{T_{n}\right\}$; but in any case, we will not make much use of the formal definition.)

Specifically we are interested in the following conjecture.

Conjecture 1. For each fixed $\Delta$ there is a $C$ such that if $T$ is any $n$-vertex tree of maximum degree at most $\Delta$, then $\mathcal{G}\left(n, C \frac{\log n}{n}\right)$ w.h.p. contains $T$.

(As usual "w.h.p." means with probability tending to 1 as $n \rightarrow \infty$.)

Of course for $p<\frac{\log n}{n}$ (we use $\log$ for $\ln$ ), $G$ is likely to contain isolated vertices, so Conjecture 1 says that the threshold for containing any bounded degree $T$ is $\Theta\left(\frac{\log n}{n}\right)$. This is known when $T$ is a Hamiltonian path [6, 13, and easy when $T$ has $\Omega(n)$ leaves (see [1,14]). It has also been proved for "almost all" trees, even without the maximum degree requirement [4]. More recently [9], it has been shown to hold with $C=1+\varepsilon$ if $T$ has $\Omega(n)$ leaves or contains a path of length $\Omega(n)$ consisting of vertices of degree 2. The best general progress to date is [14], which proves that $p \geq n^{-1+o(1)}$ suffices for all bounded degree trees, and also considers larger degrees; see this reference for some further discussion.

Conjecture 1 was proposed by the first author about twenty years ago (though stated in print only in [11], in which see also the far more general [11, Conjecture 1]), but, being a natural guess, is perhaps best considered folklore. At that early date it was also suggested that some insight might be gained by considering the case where, for some $k \mid n, T$ is the tree - here denoted Comb $b_{n, k}$

J. Kahn is supported by NSF grant DMS0701175.

N. Wormald was supported by the Canada Research Chairs Program and NSERC during this research. 
- consisting of an $(n / k)$-vertex path $P$ together with disjoint $k$-vertex paths beginning at the vertices of $P$. Such trees, which have sometimes been called "combs," may be thought of as lying somewhere between the settled cases of Conjecture 1 mentioned above.

Though we have not much non-verbal evidence, this suggestion does seem to have received quite a bit of attention, but, absent any serious progress, seems not to have produced anything in print. Here and in the companion paper [12] we establish Conjecture 1 for combs.

Theorem 1.1. There exists some fixed $C$ such that for every $n$ and $k \mid n$, the random graph $\mathcal{G}\left(n, C \frac{\log n}{n}\right)$ w.h.p. contains a copy of $\operatorname{Comb}_{n, k}$.

While this does not so far seem to be leading to a proof of Conjecture 1, it is plausible that our methods at least extend to any (bounded-degree) tree with $o(\sqrt{n})$ leaves.

The proof of Theorem 1.1 requires two entirely different arguments, depending on whether $k$ is large (at least about $\log n$ ) or small. Here we treat small $k$.

Theorem 1.2. For each $D$ there is a $K$ for which the following holds. If $k<D \log n$ divides $n$, and $v_{1}, \ldots, v_{m}$ are $m=n / k$ given (distinct) vertices, then $\mathcal{G}\left(n, K \frac{\log n}{n}\right)$ w.h.p. contains $m$ disjoint $k$-vertex paths rooted at the $v_{i}$ 's.

This is proved in Section 2, For the easy derivation of Theorem 1.1 (for small $k$ ), we may take $G=$ $G^{\prime} \cup G^{\prime \prime}$, where $G^{\prime}$ and $G^{\prime \prime}$ are independent copies of, respectively, $\mathcal{G}(n, d / n)$ for a suitable constant $d$, and $\mathcal{G}(n, p)$. (So the $p$ in Theorem 1.1 will be slightly larger than the one in Theorem 1.2.) Then $G^{\prime}$ w.h.p. contains a path $v_{1}, \ldots, v_{m}$ (assuming, as we may, that $k>1$; see, e.g., [5, Chap. 8]), which, according to Theorem 1.2, we can (w.h.p.) extend to a copy of Comb $b_{n, k}$ using $G^{\prime \prime}$.

\section{Proof of Theorem 1.2}

For a graph $H$ on $V$ and disjoint $A, B \subseteq V$, we use the notation $\nabla_{H}(A, B)=\{x y \in E(H)$ : $x \in A, y \in B\}$, omitting the subscript when $H$ is the complete graph $K_{V}$. As above, we write $G$ for $\mathcal{G}(n, p)$. Following common practice, we will sometimes pretend large numbers are integers to avoid cluttering the discussion with irrelevant floor and ceiling symbols.

Since Conjecture 1 is known to hold when $T$ has $\Omega(n)$ leaves, we may assume $k$ is at least any given constant. Though not really necessary, this will save us a little trouble in some places. Specifically we assume (as we may) that $D>2$, set

$$
\varepsilon=[D(10+\log D)]^{-1},
$$

and assume $k>2 / \varepsilon$.

Set $C=600 \varepsilon^{-1}$. With apologies, we now recycle, letting $p=C \frac{\log n}{n}$, and take our random graph $G$ to be the union of three independent copies, say $G_{1}, G_{2}, G_{3}$, of $\mathcal{G}(n, p)$. It is enough to show that $G$ w.h.p. contains the desired paths from $v_{1}, \ldots, v_{m}$ (thus giving Theorem 1.2 with $K=3 C$ ).

Set $M_{0}=W_{0}=\left\{v_{1}, \ldots, v_{m}\right\}$ and $R=V \backslash M_{0}$. It is of course enough to show

Claim 2.1. W.h.p. there is an equipartition $M_{1} \cup \cdots \cup M_{k-1}$ of $R$ such that

$$
G\left[M_{i-1}, M_{i}\right] \text { admits a perfect matching for each } i \in[k-1] \text {, }
$$


where, for disjoint $A, B \subseteq V, G[A, B]$ is the bipartite graph on $A \cup B$ with edge set $\nabla_{G}(A, B)$.

2.1. Algorithm. Set $T=\lfloor m p / 6\rfloor$ and $c=m p / T$, and note that $m p=n p / k>C / D>6000$, so $T \geq 1000$. In what follows we use $N^{i}(x)$ (respectively $N(x)$ ) for neighborhood of $x$ in $G_{i}$ (resp. $G$ ). We will show (in Section 2.2) that the following procedure w.h.p. produces a partition as in Claim 2.1.

First step: Let $\alpha \leq 1$ be a constant to be specified later and $Z=\left\{x \in R:\left|N^{1}(x) \cap W_{0}\right|<T\right\}$. Let $W_{1}, \ldots, W_{k-1}$ be disjoint random subsets of $R$ given by

$$
\mathbb{P}\left(x \in W_{i}\right)=\alpha / k \begin{cases}\forall i \in[k-1] & \text { if } x \notin Z, \\ \forall i \in\{2, \ldots, k-1\} & \text { if } x \in Z\end{cases}
$$

these choices independent for different vertices $x$. (Thus $\mathbb{P}\left(x \notin \cup W_{i}\right)$ is $1-\alpha$ or $1-\alpha(1-1 / k)$, as the case may be.) Set $W=\cup_{i=0}^{k-1} W_{i}$. The $W_{i}$ 's are our initial installments on the $M_{i}$ 's, to be augmented in the next two steps. (We won't bother with formal notation for the evolving $M_{i}$ 's.)

It will be helpful to define $L(i)=\{i-1, i+1\} \cap\{0, \ldots, k-1\}$ for $0 \leq i \leq k-1$. For $i \in[k-1]$, set

$$
B_{i}=\left\{x \in R \backslash W: \exists j \in L(i),\left|N^{1}(x) \cap W_{j}\right|<T\right\} ;
$$

these vertices will be barred from $M_{i}$. (In particular $B_{1} \supseteq Z$.)

Repair phase: For $i \in\{0, \ldots, k-1\}$ and $j \in L(i)$, let

$$
X_{i j}=\left\{x \in W_{i}:\left|N^{1}(x) \cap W_{j}\right|<T\right\} .
$$

(In particular $X_{10}=W_{1} \cap Z=\emptyset$.) We repair the $X_{i j}$ 's in some arbitrary order. Repairing $X_{i j}=\left\{x_{1}, \ldots, x_{s}\right\}$ means that for $r=1, \ldots, s$ we choose (again, arbitrarily) $T$ available vertices from $N^{2}\left(x_{r}\right)$ and add them to $M_{j}$, where a vertex is unavailable if it belongs to $B_{j}$ or has already been assigned to one of the $M_{u}$ 's. Note that the set of edges - say, $E^{2}$ — used in these "repairs" (i.e. edges from $x_{r}$ to the chosen vertices in $N^{2}\left(x_{r}\right)$ ) is a (star-)forest.

Filling in: Assign the as yet unassigned vertices to the $M_{i}$ 's so that

$$
\text { for all } i,\left|M_{i}\right|=m \text { and } M_{i} \cap B_{i}=\emptyset .
$$

The main point in all this is that, since vertices of $B_{i}$ are barred from $M_{i}$ in the repair and filling in phases, at the end of each of these phases, we have $\left|N_{G}(x) \cap W_{j}\right| \geq T$ for each $x \in M_{i}$ and $j \in L(i)$.

2.2. Analysis. We want to show that w.h.p. (i) the above procedure runs to completion and (ii) the $M_{i}$ 's produced satisfy (2.2). (It may be worth observing that $G_{3}$, which plays no role in (i), is needed for (ii).) Recalling that $\varepsilon$ was specified in (2.1), set

$$
\alpha=1 / 3, \quad \gamma=(1-3 \varepsilon) \alpha, \beta=\frac{(c \gamma-1)^{2}}{4 c \gamma}, \text { and } q=2 e^{-\beta T} .
$$

We first need some routine observations. 
Proposition 2.2. The objects produced by the first step above w.h.p. satisfy

(a) $|Z| \leq \varepsilon n$;

(b) $\left|W_{i}\right| \in(\gamma m,(1+\varepsilon) \alpha m) \forall i \in[k-1]$;

(c) $\left|B_{i}\right|<\varepsilon n \forall i \in[k-1]$;

(d) no vertex is in more than $\varepsilon k$ of the $B_{i}$ 's;

(e) $\left|X_{i j}\right|<2 m q+\log n \quad \forall i \in\{0, \ldots, k-1\}$ and $j \in L(i)$.

Of course (c) contains (a), but we state (a) first since it's needed for (b), which in turn is needed for $(\mathrm{c})$.

Note that the events in Proposition 2.2 depend only on $G_{1}$ and the $W_{i}$ 's. In fact it will be helpful to conserve some of this information: for $x \in V$ and $i \in\{0, \ldots, k-1\}$, let $\zeta(i, x)$ be the indicator of the event $\left\{\left|N^{1}(x) \cap W_{i}\right| \geq T\right\}$. Then $\{x \in Z\}=\{\zeta(0, x)=0\}(x \in R)$ and, once we have the $W_{i}$ 's, the remaining assertions in the proposition are functions of the $\zeta(i, x)$ 's.

There is nothing delicate about Proposition 2.2, and we aim for simple rather than optimal arithmetic. The following Bernstein/Chernoff-type bound (for which see e.g. [3, Lemma 8.2]) will be sufficient for our large deviation purposes. (We use $B(m, \rho)$ for a r.v. with the binomial distribution $\operatorname{Bin}(m, \rho)$.)

Lemma 2.3. For any $m, \rho$ and $t>0$,

$$
\left.\begin{array}{l}
\mathbb{P}(B(m, \rho)>m \rho+t) \\
\mathbb{P}(B(m, \rho)<m \rho-t)
\end{array}\right\}<\exp \left[-\frac{1}{4} \min \left\{t, t^{2} / m \rho\right\}\right] .
$$

Proof of Proposition 2.2. (a) For $x \in R$, we have, using Lemma 2.3,

$$
\begin{aligned}
\mathbb{P}(x \in Z) & =\mathbb{P}(B(m, p)<T) \\
& =\mathbb{P}(B(m, p)<m p-(c-1) T) \\
& <\exp \left[-\frac{(c-1)^{2}}{4 c} T\right]<q .
\end{aligned}
$$

Thus, writing " $\succ$ " for stochastic domination, we have $|Z| \prec B(n, q)$, whence, using Lemma 2.3 and $\varepsilon>2 q, \mathbb{P}(|Z|>\varepsilon n)<\exp [-(\varepsilon-q) n / 4]$.

(b) Given $Z$ satisfying (a) we have, for each $i,\left|W_{i}\right| \sim \operatorname{Bin}\left(n_{i}, \alpha / k\right)$, where $n_{1}=n-m-|Z|$ and $n_{i}=n-m$ if $i \geq 2$. In particular (for each $i$ ), $n_{i} \in((1-2 \varepsilon) n, n)$ (note $m<\varepsilon n$ because of our lower bound on $k$ ), and

$$
\begin{aligned}
\mathbb{P}\left(\left|W_{i}\right| \notin(\gamma m,(1+\varepsilon) \alpha m)\right) & \leq \mathbb{P}\left(\left|W_{i}\right| \notin\left((1-\varepsilon) \alpha n_{i} / k,(1+\varepsilon) \alpha n_{i} / k\right)\right) \\
& <2 \exp \left[-\varepsilon^{2} \alpha m / 4\right] .
\end{aligned}
$$

(c) and (d). Condition on values of $Z$ and the $W_{i}$ 's satisfying (a) and (b) - note this uses the values $\zeta(0, x)(x \in R)$ but no other information from $G_{1}$ - and write $\mathbb{P}^{\prime}$ for the corresponding conditional probabilities. (We may of course think of exposing just the edges of $G_{1}$ incident with $W_{0}$ to determine $\mathbb{P}^{\prime}$.) 
For $x \in R \backslash W$ and $i \in[k-1]$, again using Lemma 2.3, we have

$$
\begin{aligned}
\mathbb{P}^{\prime}\left(x \in B_{i}\right) & <2 \mathbb{P}(B(\gamma m, p)<T) \\
& =2 \mathbb{P}(B(\gamma m, p)<\gamma m p-(\gamma c-1) T)<q,
\end{aligned}
$$

unless $i=1$ and $x \in Z$, in which case $x$ is automatically in $B_{1}$. (If $i=1$ and $x \notin Z$, the 2's in (2.4) are unnecessary.)

Using (2.4) and independence of the events $\left\{x \in B_{i}\right\} \quad(x \in R \backslash W, i \in[k-1])$, we have (i) $\left|B_{1} \backslash Z\right|,\left|B_{2}\right|, \ldots,\left|B_{k-1}\right| \prec B(n, q)$, so that (c) holds with probability at least $1-k \exp [-(\varepsilon-q) n / 4]$, and (ii) for any $x \in R \backslash W$,

$$
\begin{aligned}
\mathbb{P}\left(\left|\left\{i \geq 2: x \in B_{i}\right\}\right| \geq\lceil\varepsilon k\rceil-1\right) & <\left(\begin{array}{c}
k \\
\lceil\varepsilon k\rceil-1
\end{array}\right) q^{\varepsilon k-1} \\
& <(e / \varepsilon)^{\varepsilon k} \exp \left[-\frac{\beta C \varepsilon}{2 c} \log n\right] \\
& <\exp \left[\left(D \log \frac{e}{\varepsilon}-\frac{\beta C}{2 c}\right) \varepsilon \log n\right]=o(1 / n) .
\end{aligned}
$$

Here we used $\left(\begin{array}{l}k \\ r\end{array}\right) \leq(e k / r)^{r} \leq(e / \varepsilon)^{\varepsilon k}$, the latter valid for $r \leq \varepsilon k ; \varepsilon k-1>\varepsilon k / 2 ; T=m p / c=$ $C \log n /(c k) ; k<D \log n$; and, for the $o(1 / n)$, the easily verified $\beta C /(2 c)-D \log (e / \varepsilon)>2 / \varepsilon$.)

(e) We retain the conditioning and notation $\mathbb{P}^{\prime}$ of $(\mathrm{c})$. We assume first that $(i, j) \neq(0,1)$ (and, since $X_{10}=\emptyset$, may also assume $\left.(i, j) \neq(1,0)\right)$. For $x \in W_{i}$ we have, as in (2.4),

$$
\mathbb{P}^{\prime}\left(x \in X_{i j}\right)<\mathbb{P}(B(\gamma m, p)<T)<q,
$$

whence $\left|X_{i j}\right| \prec B(m, q)$ and (again using Lemma 2.3)

$$
\mathbb{P}^{\prime}\left(\left|X_{i j}\right| \geq 2 m q+\log n\right)<\exp [-(m q+\log n) / 4]<n^{-1 / 4}=o(1 / k) .
$$

For $(i, j)=(0,1)$ the preceding argument is not quite applicable, since conditioning on $A:=$ $\left\{W_{1} \cap Z=\emptyset\right\}=\left\{\zeta(0, x)=1 \forall x \in W_{1}\right\}$ introduces dependencies among the edges joining $W_{0}$ and $W_{1}$. But since $A$ is an increasing event, Harris' Inequality [8] says that this conditioning does not increase the probability of the decreasing event $\left\{\left|X_{01}\right| \geq 2 m q+\log n\right\}$; so the argument in the preceding paragraph does imply $\mathbb{P}^{\prime}\left(\left|X_{01}\right| \geq 2 m q+\log n\right)=o(1 / k)$. (Of course this detail could also be dealt with by simply choosing additional random edges between $W_{0}$ and $W_{1}$.)

Write $Q$ for the intersection of the events in (a)-(e), and $S$ for the event that our process does not get stuck - that is, there are $T$ available vertices whenever the repair phase requires them and there $i s$ a way to complete the $M_{i}$ 's in the filling in phase - and the $M_{i}$ 's it produces satisfy (2.2). We have

$$
\mathbb{P}(\bar{S}) \leq \mathbb{P}(\bar{Q})+\mathbb{P}(\bar{S} \mid Q)=o(1)+\mathbb{P}(\bar{S} \mid Q),
$$

so just need $\mathbb{P}(\bar{S} \mid Q)=o(1)$.

The first part of $S$ - that the process doesn't get stuck - is easy. First, given $Q$, the number of available vertices at any repair step (at $x$ say) is at least

$$
n-m-\left(|W|+\max _{i}\left|B_{i}\right|+T \sum\left|X_{i j}\right|\right)>n / 2 .
$$


To see this notice that, since there are at most $2 k$ terms in the sum, we may bound the third term in brackets using (e) and

$$
T q=2 T e^{-\beta T} \leq 2 \frac{C}{D c} \exp \left[-\frac{C \beta}{D c}\right]<\varepsilon
$$

(say). Here the first inequality is gotten by noting that $x e^{-\beta x}$ is decreasing on $x>1 / \beta$ and that $T=m p / c \geq C /(D c)$. The second may be rewritten as

$$
\frac{1200}{c} \exp \left[-\frac{600 \beta}{\varepsilon D c}\right]<\varepsilon^{2} D
$$

which, since $c \geq 6$ and $600 \beta / c>5$ (say), follows from the easily verified

$$
200 \exp [-5(10+\log D)]<D^{-1}(10+\log D)^{-2} .
$$

We conclude that the probability that $x$ has fewer than $T$ available neighbors in $G_{2}$ is at most $\mathbb{P}(B(n / 2, p)<T)=o(1 / n)$, so that the repair phase w.h.p. finishes successfully.

Second, to say that the filling in step w.h.p. finishes successfully, it's enough to show that $Q$ implies the existence of an assignment of $M_{i}$ 's satisfying (2.3). This is a standard type of application of Hall's Theorem, briefly as follows. For $i \in[k-1]$, write $W_{i}^{*}$ for the set of vertices assigned to $M_{i}$ through the end of the repair phase, and set $W^{*}=\cup W_{i}^{*}, r_{i}=m-\left|W_{i}^{*}\right|$ and $r=\sum r_{i}=\left|R \backslash W^{*}\right|$. A set of $M_{i}$ 's with (2.3) is equivalent to a perfect matching in the bipartite graph $\Gamma$ on the vertex set $\left\{v_{i j}: i \in[k-1], j \in\left[r_{i}\right]\right\} \cup\left(R \backslash W^{*}\right)$ with $v_{i j} \sim x$ iff $x \notin B_{i}$. Then: the common size of the two sides of the bipartition is $r \in(n / 2, n)$ (see (2.6) for the lower bound); for degrees in $\Gamma$ we have $d\left(v_{i j}\right)=\left|R \backslash\left(W^{*} \cup B_{i}\right)\right|>n / 2>r / 2$ (again see (2.6) ) and, using (d), $d(x)=r-\sum\left\{r_{i}: x \in B_{i}\right\}>r-\varepsilon k m>r / 2$; and it follows easily from Hall's Theorem that a bipartite graph with $r$ vertices in each part of the bipartition and all degrees at least $r / 2$ admits a perfect matching.

We are left with the more interesting part of $S$, the assertion that (2.2) holds w.h.p. given $Q$. Say $A$ is a violator of type $(i, j, a)$ if $A \subseteq M_{i},|A|=a$, and $\left|N_{j}(A)\right|<a$, where $N_{j}(A)=N(A) \cap M_{j}$ (and $N(A)=\cup_{x \in A} N(x)$ ). By Hall's Theorem it is enough to show the following (given $Q$ ).

Claim 2.4. W.h.p. there is no violator of type $(i, j, a)$ for any $a \in\{1, \ldots,\lceil m / 2\rceil\}, i \in\{0, \ldots, k-1\}$ and $j \in L(i)$

(since if $A$ is a violator of type $(i, j, a)$ for some $a>\lceil m / 2\rceil$, then $M_{j} \backslash N_{j}(A)$ contains a violator of type $(j, i,\lceil m / 2\rceil))$.

Proof. Fix $i, j$ as in the claim and set $\vartheta=(c e)^{-2}$. We consider the cases $a \leq \vartheta m$ and $a>\vartheta m$ separately, beginning with the former.

Let $E^{1}$ be the set of edges of $G_{1}$ that meet $W$, and recall $E^{2}$ is the set of edges of $G_{2}$ that are actually used in the repair phase. If $A$ is a violator of type $(i, j, a)$, then there is some $a$-subset $B$ of $M_{j}$ containing $N_{j}(A)$. (We could, of course, require $|B|<a$.) The algorithm arranges that each vertex of $M_{i}$ is joined by $E^{1} \cup E^{2}$ to at least $T$ vertices of $M_{j}$ (we actually make no use of $\left.\left(E\left(G_{1}\right) \backslash\left(E^{1}\right)\right) \cup\left(E\left(G_{2}\right) \backslash E^{2}\right)\right)$, whence

$$
\left|\left(E^{1} \cup E^{2}\right) \cap \nabla(A, B)\right|=\left|\left(E^{1} \cup E^{2}\right) \cap \nabla\left(A, M_{j}\right)\right| \geq a T,
$$


while (since $E^{2}$ is a forest) $\left|E^{2} \cap \nabla(A, B)\right|<2 a$; so

$$
\left|E^{1} \cap \nabla(A, B)\right|>a(T-2) .
$$

Thus the probability of a violator of type $(i, j, a)$ is at most

$$
\sum_{A, B} \mathbb{P}\left(Q_{a}(A, B)\right)
$$

where $Q_{a}(A, B)$ is the event $\left\{A \subseteq M_{i}, B \subseteq M_{j},\left|E^{1} \cap \nabla(A, B)\right| \geq a(T-2)\right\}$ if $|A|=|B|=a$ and $Q_{a}(A, B)=\emptyset$ otherwise, and the sum is over $A, B \subseteq V$. (Of course if $\{i, j\} \neq\{0,1\}$ then the only nonzero summands are those with $A, B$ disjoint $a$-subsets of $R$, and, for example, when $(i, j)=(0,1)$ we are only interested in pairs with $A \subseteq W_{0}$ and $B \subseteq R$ (and $\left.|A|=|B|=a\right)$.) Note that, summing only over a-subsets $A, B$ of $V$, we have

$$
\sum_{A, B} \mathbb{P}\left(A \subseteq M_{i}, B \subseteq M_{j}\right)=\left(\begin{array}{c}
m \\
a
\end{array}\right)^{2}
$$

(since the r.v. $\sum_{A, B} \mathbf{1}_{\left\{A \subseteq M_{i}, B \subseteq M_{j}\right\}}$ is actually the constant $\left(\begin{array}{c}m \\ a\end{array}\right)^{2}$; of course by symmetry the summand in (2.8) is the same for all $(A, B)$ of interest, but we don't need this).

On the other hand, we will show (provided the conditioning event is not vacuous)

$$
\mathbb{P}\left(Q_{a}(A, B) \mid A \subseteq M_{i}, B \subseteq M_{j}\right)<(1-q)^{-2 a}\left(\begin{array}{c}
a^{2} \\
a(T-2)
\end{array}\right) p^{a(T-2)} .
$$

Given this we just need a little arithmetic: the combination of (2.8) and (2.9) yields

$$
\begin{aligned}
\sum_{A, B} \mathbb{P}\left(Q_{a}(A, B)\right) & \leq\left(\begin{array}{c}
m \\
a
\end{array}\right)^{2}(1-q)^{-2 a}\left(\begin{array}{c}
a^{2} \\
a(T-2)
\end{array}\right) p^{a(T-2)} \\
& \leq\left[(1-q)^{-2}\left(\frac{e m}{a}\right)^{2}\left(\frac{e a p}{T-2}\right)^{T-2}\right]^{a} \\
& =\left[\left(\frac{e}{1-q}\right)^{2}\left(\frac{a}{m}\right)^{T-4}\left(\frac{e m p}{T-2}\right)^{T-2}\right]^{a} \\
& <\left[(c e)^{T}\left(\frac{a}{m}\right)^{T-4}\right]^{a}
\end{aligned}
$$

(say), which easily implies

$$
\sum_{a=1}^{\lfloor\vartheta m\rfloor} \sum_{A, B} \mathbb{P}\left(Q_{a}(A, B)\right)<O(1 / m)^{T-4} .
$$

It remains to prove (2.9). Here it is helpful to think of our procedure as choosing

(i) $\zeta(0, x)$ for $x \in R$, thus specifying $Z$;

(ii) $W_{1}, \ldots, W_{k-1}$;

(iii) $\zeta(i, x)$ for $i \in[k-1]$ and $x \in V \backslash W=: Y$, thus specifying the $B_{i}$ 's 
(and then continuing). It is then evident that the only information from $E\left(G_{1}\right)$ with any bearing on our choices of the sets $W_{l}$ and $M_{l} \backslash W_{l}$ is that in (i) and (iii); in particular, we have the following.

Observation 2.5. The pair $\left(M_{i}, M_{j}\right)$, set $E^{1} \cap \nabla\left(W_{i} \cup W_{j}, Y\right)$ and indicators $\mathbf{1}_{\left\{x y \in E^{1}\right\}}$ for $(x, y) \in$ $W_{i} \times W_{j}$ are conditionally (mutually) independent given $W_{i}, W_{j}$ and the values of $\zeta(i, x)$ and $\zeta(j, x)$ for $x \in Y$.

Suppose now that we're given $W_{i}, W_{j}, M_{i}, M_{j}$ with $A \subseteq M_{i}$ and $B \subseteq M_{j}$. For a set $X$ we use $B(X, p)$ for the distribution on the power set of $X$ that assigns $U \subseteq X$ probability $p^{|U|}(1-p)^{|X \backslash U|}$.

We assume first that we are not in one of the slightly special cases with $\{i, j\}=\{0,1\}$. According to Observation 2.5, the sets $E^{1} \cap \nabla\left(x, W_{i}\right)$ and $E^{1} \cap \nabla\left(x, W_{j}\right)(x \in Y)$ and the indicators $\mathbf{1}_{\left\{x y \in E^{1}\right\}}$ $\left(x \in W_{i}, y \in W_{j}\right)$ are mutually independent. Each of the indicators is Bernoulli with mean $p$; each $E^{1} \cap \nabla\left(x, W_{i}\right)$ is distributed as $\mathbf{F}:=B\left(\nabla\left(x, W_{i}\right), p\right)$ conditioned on $\{|\mathbf{F}| \geq T\}$, an event of probability at least $\mathbb{P}(\operatorname{Bin}(\gamma m, p) \geq T)>1-q$ (see Proposition 2.2(b) and (2.4)); and similarly for the $E^{1} \cap \nabla\left(x, W_{j}\right)$ 's. Thus we can bound the probability of any event determined by $E^{1} \cap \nabla(A, B)$, by computing its probability assuming all edges occur independently with probability $p$, and then multiplying by $(1-q)^{-\left|A \backslash W_{i}\right|+\left|B \backslash W_{j}\right|} \leq(1-q)^{-2 a}$. This gives (2.9):

$$
\begin{aligned}
\mathbb{P}\left(Q_{a}(A, B) \mid A \subseteq M_{i}, B \subseteq M_{j}\right) & <(1-q)^{-2 a} \mathbb{P}\left(\operatorname{Bin}\left(a^{2}, p\right) \geq a(T-2)\right) \\
& <(1-q)^{-2 a}\left(\begin{array}{c}
a^{2} \\
\lfloor a(T-2)\rfloor
\end{array}\right) p^{a(T-2)}
\end{aligned}
$$

When $\{i, j\}=\{0,1\}, Q_{a}(A, B)$ is determined by the sets $E^{1} \cap \nabla\left(x, W_{0}\right)$, for $x \in A$ if $i=1$ and $x \in B$ if $i=0$. Recalling that the choice of $M_{1}$ depends only on $\zeta(0, x)$ for $x \in R$, these sets are independent, each distributed as $\mathbf{F}:=B\left(\nabla\left(x, W_{0}\right), p\right)$ conditioned on $\{|\mathbf{F}| \geq T\}$, an event of probability at least $\mathbb{P}(B(m, p) \geq T)>1-q$, and (2.9) follows as before. (In this case $(1-q)^{-2 a}$ could be replaced by $(1-q)^{-a}$.)

For the simpler analysis when $a>\vartheta m$ (and $a \leq\lceil m / 2\rceil$ ), we just use $G_{3}$. Here a violator $A$ of type $(i, j, a)$ satisfies $\nabla(A, B)=\emptyset$ for some $B \subseteq M_{j}$ of size $\lceil m / 2\rceil$; so the probability of such a violator is less than

$$
\sum_{A, B} \mathbb{P}\left(A \subseteq M_{i}, B \subseteq M_{j}, E\left(G_{3}\right) \cap \nabla(A, B)=\emptyset\right)<4^{m}(1-p)^{a m / 2}=o(1),
$$

where the sum is over disjoint $A, B \subseteq V$ (but really, for example, over $A, B \subseteq R$ unless $\{i, j\}=$ $\{0,1\})$ with $|A|=a$ and $|B|=\lceil m / 2\rceil$, and we used

$$
\begin{gathered}
\sum_{A, B} \mathbb{P}\left(A \subseteq M_{i}, B \subseteq M_{j}\right)=\left(\begin{array}{c}
m \\
a
\end{array}\right)\left(\begin{array}{c}
m \\
\lceil m / 2\rceil
\end{array}\right), \\
\mathbb{P}\left(E\left(G_{3}\right) \cap \nabla(A, B)=\emptyset \mid A \subseteq M_{i}, B \subseteq M_{j}\right) \leq(1-p)^{a m / 2}
\end{gathered}
$$

(of course here $G_{3}$ is actually independent of the conditioning), and (recalling $k<D \log n$ ) $\vartheta m p / 2 \geq$ $\vartheta C /(2 D)>2 \log 4$. 


\section{REFERENCES}

[1] N. Alon, M. Krivelevich, and B. Sudakov, Embedding nearly-spanning bounded degree trees, Combinatorica 27 (2007), no. 6, 629-644.

[2] N. Alon and J. H. Spencer, The probabilistic method, 3rd ed., John Wiley \& Sons Inc., 2008.

[3] J. Beck and W. W. L. Chen, Irregularities of distribution, Cambridge Tracts in Mathematics, vol. 89, Cambridge University Press, Cambridge, 1987.

[4] E. A. Bender and N. C. Wormald, Random trees in random graphs, Proc. Amer. Math. Soc. 103 (1988), no. 1, 314-320.

[5] B. Bollobás, Random graphs, 2nd ed., Cambridge Studies in Advanced Mathematics, vol. 73, Cambridge University Press, Cambridge, 2001.

[6] B. Bollobás, The evolution of sparse graphs, Graph theory and combinatorics (Cambridge, 1983), Academic Press, London, 1984, pp. 35-57.

[7] P. Erdős and A. Rényi, On the evolution of random graphs, Publ. Math. Inst. Hungar. Acad. Sci. 5 (1960), $17-61$.

[8] T. E. Harris, A lower bound for the critical probability in a certain percolation process, Proc. Cambridge Philos. Soc. 56 (1960), 13-20.

[9] D. Hefetz, M. Krivelevich, and T. Szabó, Sharp threshold for the appearance of certain spanning trees in random graphs, Random Structures Algorithms 41 (2012), no. 4, 391-412.

[10] S. Janson, T. Łuczak, and A. Rucinski, Random graphs, Wiley-Interscience Series in Discrete Mathematics and Optimization, Wiley-Interscience, New York, 2000.

[11] J. Kahn and G. Kalai, Thresholds and expectation thresholds, Combin. Probab. Comput. 16 (2007), no. 3, 495-502.

[12] J. Kahn, E. Lubetzky, and N. Wormald, Cycle factors and renewal theory, preprint.

[13] J. Komlós and E. Szemerédi, Limit distribution for the existence of Hamiltonian cycles in a random graph, Discrete Math. 43 (1983), no. 1, 55-63.

[14] M. Krivelevich, Embedding spanning trees in random graphs, SIAM J. Discrete Math. 24 (2010), no. 4, 14951500 .

[15] N. C. Wormald, Models of random regular graphs, Surveys in combinatorics, 1999 (Canterbury), London Math. Soc. Lecture Note Ser., vol. 267, Cambridge Univ. Press, Cambridge, 1999, pp. 239-298.

JEFF KAHN

Department of Mathematics, Rutgers, Piscataway, NJ 08854, USA.

E-mail address: jkahn@math.rutgers.edu

EYAL LUBETZKY

Microsoft Research, One Microsoft Way, Redmond, WA 98052, USA.

E-mail address: eyal@microsoft.com

Nicholas Wormald

School of Mathematical Sciences, Monash University, Clayton, Victoria 3800, Australia.

E-mail address: nick.wormald@monash.edu 